\title{
Finite elements for thixotropic elasto-viscoplastic flows
}

\author{
Sérgio Frey, $\quad$ Fernanda B. Link, Márleson R. S. Ferreira \\ Departament of Mechanical Engineering, UFRGS, \\ 90050-170-000, Porto Alegre, RS \\ E-mail: frey@mecanica.ufrgs.br, feulink@mecanica.ufrgs.br, marleson@mecanica.ufrgs.br
}

\author{
Mônica F. Naccache, Paulo R. de Souza Mendes, \\ Departament of Mechanical Engineering, PUC-Rio, \\ 22453-900, Rio de Janeiro, RJ \\ E-mail: naccache@puc-rio.br, pmendes@puc-rio.br
}

\begin{abstract}
This work aims at performing finite element approximations for entry flows of thixotropic elasto-viscoplastic fluids, using the structured fluid model introduced in de Souza Mendes (2011). The constitutive equation is based on a modification of the Oldroyd-B viscoelastic equation, with variable viscosity and relaxation time. According to this model, both the relaxation time and the viscosity are functions of a scalar parameter - denoted herein by structure parameter - that deals with microscopic changes in the material microstructure. All results are obtained using a four-field GLS-type method, aiming at determining the accurate morphology and position of the unyielded regions in entry flows of elasto-viscoplastic fluids through an one-to-four sudden expansion.
\end{abstract}

Keyword: Thixotropy, elasto-viscoplasticity, entry flow, multi-field GLS method.

\section{Introduction}

Thixotropic fluids are present in relevant industrial applications as drilling fluids in the oil industry, cosmetic in the pharmaceutic industry and polymeric solutions in the plastic processing (Bernes, 1997). These fluids are compounds formed by macromolecules, which confer them a microscopic structure that influences their macroscopic response to an applied stress (Mewis and Wagner, 2009).

In this article, finite element solutions were obtained for the flow of incompressible thixotropic elasto-viscoplastic materials through a one-to-four sudden expansion. The mechanical behavior of the material is described by a recent constitutive model the extra-stress equation of which is a modified Oldroyd-B model possessing structuring-level dependent viscosity, relaxation time and retardation time. The local structuring level is given by the structure parameter, a scalar-valued field that is governed by a kinetics-type evolution equation (Mujumdar et al., 2002).

The differential equations of the constitutive model are integrated numerically in conjunction with the mass and momentum balance equations using a multi-field Galerkin least-squares (GLS) method that employs as primal variables the structure parameter $\lambda$, the extra-stress $\boldsymbol{\tau}$ and the fluid velocity $\boldsymbol{u}$ and the isotropic pressure $p$. Since the GLS method is immune to the BabuskaBrezzi stability condition, the computational domain in partitioned into equal-order (bi-linear) Lagrangian finite elements, a very attractive feature from the computational point of view.

The influence of the thixotropic equilibrium time, elastic modulus, yield stress and flow intensity on the fields of velocity and pressure, as well as on the fields of the intensities of 
stress and strain rate is investigated for relevant ranges of these parameters. The field of elastic strain in both the yielded and unyielded regions is also given, as well as the topology of the yielded surfaces. The results show that thixotropy postpones the buildup of the microstructure to further downstream locations. Elasticity causes a time delay of the microstructure breakup of the unyielded material that leaves the plug-flow region of the upstream channel, crosses the expansion plane (where high extensional stresses occur) and enters the downstream channel.

\section{Mathematical modeling}

The conservation equations of mass and momentum, and the constitutive equation proposed by de Souza Mendes (2011) to model a thixotropic elasto-viscoplastic fluid are given by:

$$
\begin{array}{rrl} 
& \partial_{x_{k}} u_{k}=0 & \text { in } \Omega \\
\partial_{x_{i}} \mathcal{P}-\partial_{x_{j}} \tau_{i j}-2 \eta_{\infty} \partial_{x_{j}} D(u)_{i j}=0 \text { for } i=1, \cdots N & \text { in } \Omega \\
\tau_{i j}+\theta(\lambda) \check{\tau}_{i j}-2 \eta(\lambda) D(u)_{i j}=0 \text { for } i, j=1, \cdots N & \text { in } \Omega
\end{array}
$$

where $\check{\tau}_{i j}$ stands for the upper-convected derivative of the extra-stress tensor,

$$
\check{\tau}_{i j}=u_{k} \partial_{x_{k}} \tau_{i j}-\partial_{x_{k}} u_{i} \tau_{k j}-\tau_{i k} \partial_{x_{k}} u_{j} \quad \text { in } \Omega
$$

The relaxation time $\theta$, the elastic modulus $G$ and the structural viscosity $\eta$ are functions of the structure parameter $\lambda$ that maps the material structuring level,

$$
\theta(\lambda)=\frac{\eta(\lambda)}{G(\lambda)} \quad ; \quad G(\lambda)=G_{0} e^{m\left(\frac{1}{\lambda}-1\right)} \quad ; \quad \eta(\lambda)=\left(\frac{\eta_{0}}{\eta_{\infty}}\right)^{\lambda} \eta_{\infty}
$$

with $G_{0}$ representing the elastic modulus of a fully-structured material and $\lambda$ determined by the evolution equation below,

$$
u_{k} \partial_{x_{k}} \lambda=\frac{1}{t_{e q}}\left[(1-\lambda)-\left(1-\lambda_{e q}\right)\left(\frac{\lambda}{\lambda_{e q}}\right)\left(\frac{\tau}{\eta(\lambda) \dot{\gamma}}\right)\right]
$$

The equilibrium-state of the structure parameter can be obtained from the equilibrium viscosity,

$$
\lambda_{e q}(\dot{\gamma})=\frac{\ln \eta_{e q}(\dot{\gamma})-\ln \eta_{\infty}}{\ln \eta_{0}-\ln \eta_{\infty}}
$$

where $\eta_{e q}$ is given by the SMD viscoplastic viscosity function, proposed in de Souza Mendes and Dutra (2004),

$$
\eta_{e q}(\lambda)=\left[1-\exp \left(-\frac{\eta_{0} \dot{\gamma}}{\tau_{0}}\right)\right]\left[\frac{\tau_{0}-\tau_{0_{d}}}{\dot{\gamma}} \exp \left(-\frac{\dot{\gamma}_{0}}{\dot{\gamma}_{0_{d}}}\right)+\frac{\tau_{0_{d}}}{\dot{\gamma}}+K \dot{\gamma}^{n-1}\right]+\eta_{\infty}
$$

$\eta_{0}$ and $\eta_{\infty}$ are the viscosity of fully structured and unstructured fluids, and $\tau_{0}$ and $\tau_{0_{d}}$ are the static and dynamic yield stress.

\section{$3 \quad$ Numerical Results}

A four-field GLS-type formulation (Behr, et al. 1993), in terms of the structure parameter $\lambda$, extra-stress tensor $\boldsymbol{\tau}$, the pressure field $p$ and the velocity vector $\mathbf{u}$, is employed to approximate thixotropic elasto-viscoplastic fluids flowing through an one-to-four sudden expansion. Thanks of the good convergence features of the GLS formulation - see, for instance, Franca and Frey, 1992; Behr, et al. (1993); and references therein - the computations herein performed make 
use of equal-order combination of Lagrangian bi-linear $\left(Q_{1}\right)$ finite element interpolations as well as generate stable stable approximation for thixotropic- and elastic-dominate flows. The nonlinear system of equations resulted from the discretization of GLS formulation is solved by a quasi-Newton method. This algorithm makes use of a frozen Jacobian gradient strategy, only updating the Jacobian matrix at each two or three quasi-Newtonian iterations.

Fig. 1 presents the problem statement of the studied geometry, for a system of Cartesian coordinate fixed at the inferior inlet section of the small channel. The aspect ratio $\theta$, relating the small $(h)$ and large $(H)$ heights, is maintained constant as $\beta=0.25$ in all computations. The flow is assumed to have a plane of symmetry along its center-line $\left(x_{2}=0\right)$ and, therefore, only one half of the domain is considered. In order to assure fully-developed flows in both small and large channels, the length of the small channel (l) is fixed as thirty times its height $(h)$, while the length of the large channel $(L)$ is forty five times the height of its height.

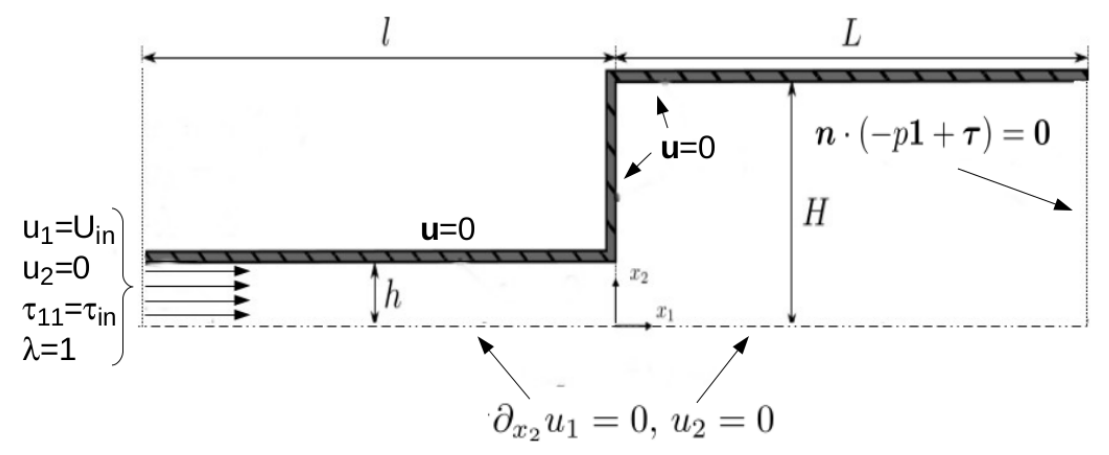

Figura 1: The sudden expansion flow: the problem statement.

The kinematics boundary conditions are assumed to be: (i) no-slip and impermeability $\left(u_{1}=u_{2}=0\right)$ along the channel wall; (ii) symmetry $\left(\partial_{x_{2}}\left(u_{1}\right)=u_{2}=\tau 12=0\right)$ along the channel center-line ; (iii) uniform velocity $\left(u_{1}=U ; u_{2}=0\right)$ and extra-stress $\left(\tau_{12}=\tau_{11}=\tau_{12}=0\right.$ ) profiles at the channel inlet; (iv) free-traction $\left(-p+2 \eta D_{11}=-p+2 \eta D_{21}=0\right)$ at the channel outlet. For the structure parameter, the following boundary conditions are imposed: unitary profile $(\lambda=1)$ along the channel inlet; (v) null gradients of lambda $\left(\partial_{x_{i}}(\lambda)=0\right)$ along the other boundaries.

The dimensionless governing parameter arised from the dimensioless form (de Souza Mendes, 2007) of flow governing equations are the flow rate $U^{*}$ - derived from the velocity boundary condition,

$$
U^{*}=\frac{U_{i n}}{\dot{\gamma}_{1} h}=\frac{K^{1 / n} U_{i n}}{\tau_{y}^{1 / n} h}=\left(\frac{K U_{i n}^{n}}{\tau_{y} h^{n}}\right)^{1 / n}=\frac{1}{H B^{1 / n}}
$$

the characteristic time of the microstructing change $t_{e q}^{*}$ - obtained from evolution equation and controls the thixotropic level,

$$
t_{e q}^{*}=t_{e q} \dot{\gamma}_{1}
$$

and the relaxation time of the fully-structure fluid $(\lambda=1)$ - came out from the extra stress viscoelastic equation and it is a measure of the elasticity level,

$$
\theta_{0}^{*}=\theta_{0}^{*} 1=\frac{\eta_{0} \dot{\gamma}_{1}}{G_{0}}=\frac{\tau_{y} \dot{\gamma}_{1}}{G_{0}}(J+1)
$$

The effect of the flow intensity on flow pattern is illustrated in Fig. 2 for the dimensionless lid velocity $U^{*}$ from 0.1 to 0.7 . It is noted that for larger values of $U^{*}$, the unyielded regions are smaller, due to the higher flow intensity imposed, which implies higher stress and strain-rate levels in the flow field. These regions are located close to the centerline of both channels, where 
the fluid flows in a nearly rigid body motion and at the expansion corner, where the fluid is almost quiescent.

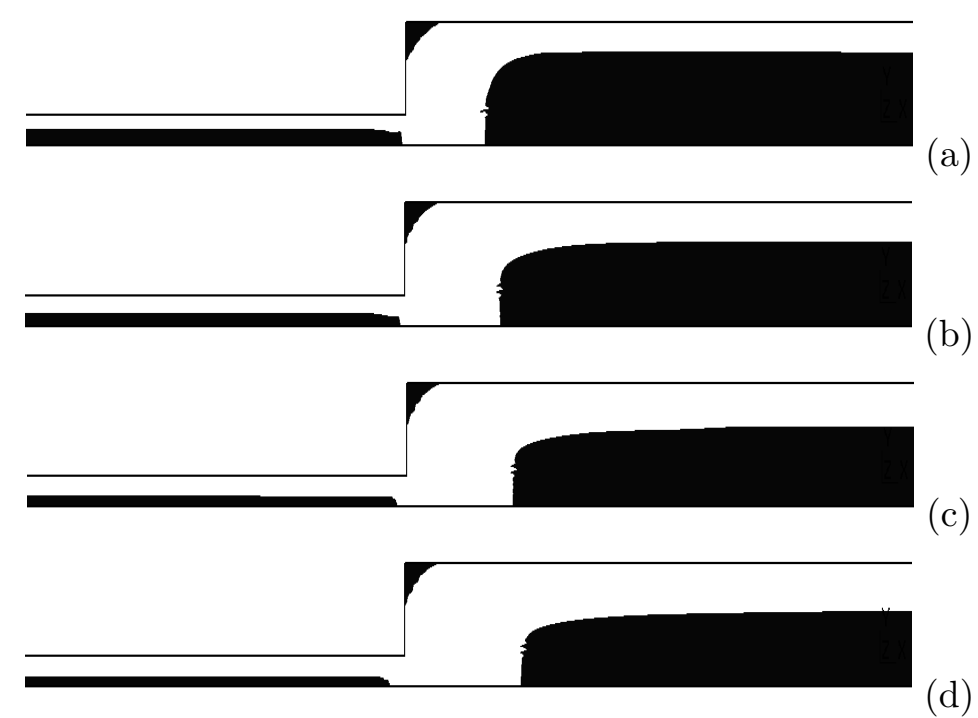

Figura 2: Unyielded regions: $\tau^{*}$-isobands for $\theta_{0}^{*}=833, t_{e q}^{*}=1.0, \eta_{0}^{*}=10^{4}, \eta_{\infty}^{*}=10^{-2}, n=$ $0.5, m=2.0$ : (a) $U^{*}=0.1$; (b) $U^{*}=0.3$; (c) $U^{*}=0.5$; (d) $U^{*}=0.7$.

In Fig. 3 the thixotropy influence is illustraded through the ranging of $t_{e q}^{*}$ from 1.0 to 7.0. It can be observed from this set of figures that, after the expansion region, particles go downstream unstructured, traveling longer and longer distances until to reestablish their equilibrium. In the Eulerian point of view, the increasing of $t_{e q}^{*}$ leads that the structuring level is convected farther and farther downstream the expansion plane.

We finally examine the effect of elasticity on the structuring level and the shape and location of the unyielded regions. To this end, Figure 4 gives the isobands of both $\lambda$ and $\tau$ distributions, for asymptotic values of the dimensionless stiffness $\theta_{0}^{*}$, namely 100 and 909 . This couple of figures essencially shows that elasticity increase leads to the plug-flow of the smaller channel invade the larger one due to memory effects.

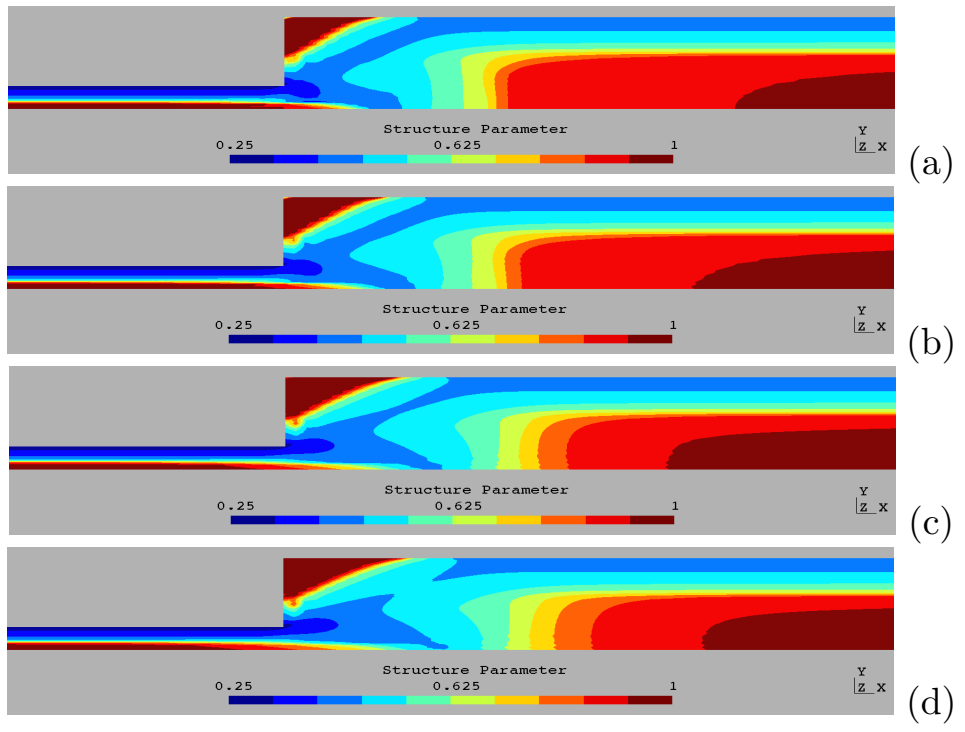

Figura 3: Thixotropy level: $\lambda^{*}$-isobands for $U^{*}=0.1, \theta_{0}^{*}=833, \eta_{0}^{*}=10^{4}, \eta_{\infty}^{*}=10^{-2}, n=$ $0.5, m=2.0$ : (a) $t_{e q}^{*}=1.0$; (b) $t_{e q}^{*}=2.0$; (c) $t_{e q}^{*}=5.0$; (d) $t_{e q}^{*}=7.0$. 


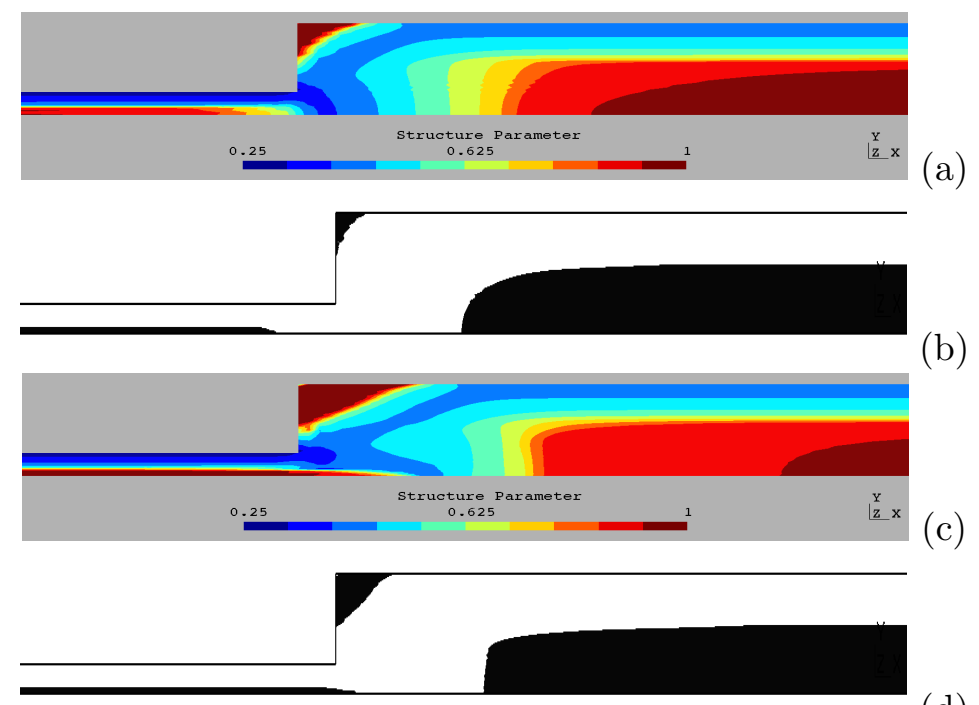

(d)

Figura 4: Elasticity level: $\lambda^{*}$ - and $\tau$-isobands for $U^{*}=0.1, t_{e q}^{*}=1.0, \eta_{0}^{*}=10^{4}, \eta_{\infty}^{*}=10^{-2}, n=$ $0.5, m=2.0$ : (a) $\theta_{0}^{*}=100$; (b) $\theta_{0}^{*}=100$; (c) $\theta_{0}^{*}=909$; (d) $\theta_{0}^{*}=909$.

\section{Final Remarks}

This work undertook finite element approximations for thixotropic elasto-viscoplastic fluids flowing through a one-to-four sudden planar contraction. In addition to usual balance equations of mass and momentum, the mechanical model used to describe the material behavior (as proposed by de Souza Mendes (2011) was made up of an Oldroyd-B-like equation for the extra-stress tensor coupled with an evolution equation for the structure parameter. The mechanical model was approximated making use of a four-field GLS-type formulation in terms of the structure parameter, extra-stress tensor, the pressure and velocity fields. The computational domain was partitioning into a bi-linear Lagrangian finite element mesh for all primal variables. The simulations performed intended to analyze the effect of elasticity, viscous and thixotropic effects on the flow pattern. All simulations attested the relevance of the right determination of the structuring level on accurate computation of the shape end location of unyielded regions, along with the strong influence of elasticity on the topology of yield surfaces. .

\section{Acknowledgments}

The authors acknowledge Petrobras S.A., MCTI/CNPq, CAPES, FAPERJ and FINEP for financial support.

\section{References}

A. Mujumdar, A. N. Beris, A. B. Metzner, Transient phenomena in thixotropic systems, J. Non-Newtonian Fluid Mech., 102 (2002) 157-178.

H. A. Barnes, Thixotropy - a review , J. Non-Newtonian Fluid Mech, 70 (1997) 1-33.

J. Mewis, N. J. Wagner, Thixotropy, Adv Colloid Interface Sci, 147 (2009) 214-227.

L. P. Franca , S. L. Frey, Stabilized finite element methods: II. The incompressible NavierStokes equations, Comput. Meth. Appl. Mech. Eng., 99 (1992) 209-233. 
M. A. Behr, L. P. Franca, T. E. Tezduyar, Stabilized finite element methods for the velocitypressure-stress formulation of incompressible flows, Comput. Methods Appl. Mech. Engrg, 104 (1993) 31-48.

P.R. De Souza Mendes, Thixotropic elasto-viscoplastic model for structured fluids, Comput. Methods Appl. Mech. Engrg, 7 (1993) 2471-2483.

P.R. De Souza Mendes, Dimensionless non-Newtonian fluid mechanics, Journal of Non-Newtonian Fluid Mechanics, 147 (1993) 109-116. 